Bibliotecas. Vol 38, № 1, enero - junio, 2020. EISSN: 1659-3286

URL: http://www.revistas.una.ac.cr/index.php/bibliotecas/index

DOI: http://dx.doi.org/10.15359/rb.38-1.1

Licencia: Creative Commons (BY-NC-SA) 4.0 Internacional

\title{
Propuesta de un Sistema Integrado de Gestión Bibliotecaria para el Sistema de Bibliotecas Municipales de la Municipalidad de San José
}

\author{
Proposal for an Integrated Library Management System for the Municipal Library \\ System of the Municipality of San José
}

\author{
Máster Jenny Ulate Montero \\ Universidad Nacional, Costa Rica
}

Recibido: 01 de febrero 2019

Aceptado: 06 de junio de 2019

Publicado: 12 de marzo de 2020

\section{Resumen}

En este estudio se describe el estado actual del proceso de automatización de las bibliotecas municipales del cantón Central de San José, con el fin de proponer el Sistema Integrado de Gestión Bibliotecaria como herramienta tecnológica necesaria para llevar a cabo dicho proceso. Se realizó un diagnóstico que permitió, entre otros aspectos: describir los procesos bibliotecarios, el estado actual de los servicios y de los productos de información de las bibliotecas municipales; describir los recursos humanos, financieros, bibliográficos y de infraestructura tecnológica con los que cuentan estas bibliotecas en función de los requerimientos funcionales del Sistema Integrado de Gestión Bibliotecaria para la automatización de las bibliotecas municipales; identificar la factibilidad técnica, económica y operacional de dicho sistema; y evaluar diferentes programas de automatización para proponer el sistema que permita automatizar el Sistema de Bibliotecas Municipales.

\section{Palabras clave:}

Bibliotecas Municipales, Sistemas Integrados de Gestión Bibliotecaria, Automatización de Bibliotecas.

\begin{abstract}
This project describes the automation process for the Municipal Libraries of the Central canton of San Jose with the purpose of proposing an Integrated Library Management System that pretends to solve the need of having the technological tool to carry out this process. A diagnose was made with the intent to describe the library processes and the current state of the products and services of the municipal libraries. It also includes a description of the different resources the libraries have, such as human, financial and technical infrastructure, in order to determine the functional requirements for the Integrated System proposed so we can identify the technical, economical and operational feasibility to implement the appropriate automation program for the Municipal Libraries System.
\end{abstract}

\section{Keywords:}

Municipal Libraries, Integrated Library Management Systems, Automation of Libraries. 


\section{Introducción}

La sociedad actual se caracteriza por demandar grandes producciones y flujos de información. El lugar que ocupa está en las diferentes actividades humanas ha alcanzado niveles de uso inadvertidos en años anteriores. En este contexto, surge la sociedad de la información y del conocimiento caracterizada por el uso de nuevas tecnologías de información y comunicación que constituyen un agente de cambio en todos los aspectos de la vida, así como en las estructuras sociales, en el comercio y en los mercados nacionales e internacionales.

Con el surgimiento de la sociedad de la información, las relaciones sociales, económicas, el trabajo, la cultura y la comunicación han cambiado. Masuda, mencionado por Saorín (2002) indica que la sociedad de la información es "aquella que crece y se desarrolla alrededor de la información". (p. 17). Esta realidad, producto del avance tecnológico, representa para las instituciones públicas y privadas un verdadero desafío. Particularmente, la tecnología ha tenido un fuerte impacto en las bibliotecas, al inicio, con los procesos de automatización y, luego, con nuevas formas de comunicación en espacios web y redes sociales, lo que impone un nuevo modelo de bibliotecas.

Al respecto, Valdés $(2007$, p. 1) menciona la decisión de la Federación Internacional de Asociaciones de Bibliotecarios (IFLA) sobre "el nuevo papel que deberán asumir las bibliotecas y los bibliotecarios ante la sociedad de la información y el conocimiento", indicando que la concepción bibliotecaria se fundamenta en pilares, tales como la organización en el contexto social, las bibliotecas y los servicios de información en consideración a tres factores esenciales: sociedad, profesión y miembros.

Además, Valdés (2007) también señala la reunión del 2003 en Ginebra, Suiza, en la que la IFLA y la Organización de las Naciones Unidas para la Educación, la Ciencia y la Cultura (UNESCO) promueven los "principios de libre acceso a la información y expresión sin restricciones y se exhorta para que los individuos sean capaces de crear, acceder, usar y compartir información y conocimiento para la sociedad" (p.1). Lo anterior evidencia la evolución de las distintas actividades y tareas de las bibliotecas y de los servicios que se diseñan. El comercio electrónico, 
la educación virtual, el acceso a fondos bibliográficos y a bases de datos digitales redefinen la función y los servicios de las bibliotecas en relación con la gestión estratégica de las tecnologías de información y comunicación. Por otra parte, están las demandas cada vez más exigentes de la población usuaria de las bibliotecas y de procesos administrativos cada vez más complejos, razón por la cual es necesario ofrecer mecanismos que permitan aplicar las nuevas tecnologías en los procesos bibliotecarios, mediante la automatización.

En este contexto tecnológico, en el que las bibliotecas se convierten en verdaderos sistemas de gestión del conocimiento e información, cuyos elementos, tareas, procesos y servicios se interrelacionan, se consideró necesario analizar la situación actual de la automatización del Sistema de Bibliotecas Municipales de la Municipalidad del cantón Central de San José. Estas bibliotecas están adscritas a la Gerencia de Provisión de Servicios y al Departamento de Servicios Culturales. Son espacios de trabajo encargados de diseñar programas y ejecutar servicios de extensión cultural. Actualmente, lo conforman siete bibliotecas, ubicadas en distintos distritos del cantón Central de San José. Estas son instituciones socioculturales, desarrolladoras de acciones encaminadas a conseguir mejores condiciones personales y sociales de los habitantes del cantón Central de San José. Desde el 2005, las bibliotecas municipales iniciaron un proceso de transformación, cuyo objetivo principal fue convertir las bibliotecas infantiles en bibliotecas municipales con mayor cobertura de usuarios y servicios.

Respecto a la organización y normalización de la información y de los procesos técnicos, estas bibliotecas contaban únicamente con catálogos manuales, los cuales permitían tener acceso, mediante fichas catalográficas, al material existente en cada biblioteca. En el 2000, se diseñó una base de datos referencial, elaborada en el programa Winisis. Posteriormente, en el 2008, surge el proyecto de crear e implementar un Centro Catalográfico, unidad que tiene a cargo el procesamiento técnico centralizado de los materiales bibliográficos provenientes de las siete bibliotecas, además de mejorar una serie de tareas técnicas y administrativas ejecutadas por el personal de las bibliotecas, relacionadas con la correcta organización, normalización y centralización de los procesos técnicos. 
La base de datos disponible reunía las colecciones de las siete bibliotecas y, desde la creación del Centro Catalográfico, se desarrolló de acuerdo con normas internacionales y homogeneidad de los criterios y principios de catalogación; sin embargo, no era una base de datos integrada, lo que limitaba brindar servicios como acceso al catálogo público en línea para consulta de los usuarios. Desde sus inicios, estas bibliotecas han prestado sus servicios de forma manual y con el uso de controles que no permitían una gestión ágil y oportuna, evidenciándose, en algunas ocasiones, duplicidad de procesos, datos estadísticos inexactos y elaboración de procesos arduos y complejos.

Esta situación hizo necesario analizar el grado de avance del proceso de automatización del sistema de bibliotecas municipales, ya que estas bibliotecas no contaban con un Sistema Integrado de Gestión Bibliotecaria (SIGB), lo que dificultaba facilitar a toda la colectividad social el acceso ágil y rápido a la información y material existente en las siete bibliotecas. Se consideró que, al automatizar los servicios y diferentes procesos, mediante la implementación de un SIGB, el servicio que brindan estas bibliotecas a las comunidades sería más oportuno, inclusive abriría las puertas a nuevos servicios y nueva población usuaria.

El propósito de implementar un SIGB fue establecer un nuevo modelo de bibliotecas municipales, capaces de gestionar la información y el conocimiento, como valor fundamental de desarrollo local e integrar todas las funciones y servicios de las bibliotecas, minimizar tiempos y costos en la gestión de las bibliotecas, reducir tiempos de respuestas a las demandas de los usuarios, ofrecer herramientas para que los habitantes del cantón Central de San José (niños y niñas, jóvenes, adultos, adultos mayores, personas y grupos con discapacidad, organizaciones comunales, grupos educativos, culturales, sector empresarial, Gobierno y administración local y organizaciones nacionales e internacionales) puedan recuperar información de manera rápida, precisa, completa y uniforme.

La propuesta de un SIGB abordó la situación del rezago tecnológico en el manejo de la información en las siete bibliotecas de la Municipalidad de San José. La implementación del SIGB supuso una mejora en el funcionamiento de las bibliotecas municipales, el cual permitiría tener controles más adecuados de los materiales bibliográficos existentes, rapidez en las 
consultas, precisión en el registro de la información y, sobre todo, mayores beneficios para los usuarios, además de mejorar el acceso a los recursos de información disponibles en las bibliotecas municipales del cantón Central de San José.

\section{Objetivos}

Al investigar la situación actual en el proceso de automatización de las bibliotecas municipales y proponer la implementación de un Sistema Integrado de Gestión Bibliotecaria (SIGB), surgieron algunas interrogantes en torno a dicho proceso: ¿cómo debe realizarse el proceso de automatización?, ¿cuál es el nivel de automatización que requerían las bibliotecas municipales? y ¿qué procesos técnicos- administrativos y de servicios deben automatizarse? A partir de estas inquietudes se plantearon los siguientes objetivos:

\section{Objetivo general 1}

Realizar un diagnóstico sobre el estado actual del proceso de automatización de las Bibliotecas Municipales del cantón Central de San José.

\section{Objetivos específicos}

1. Describir la situación actual del proceso de automatización en las bibliotecas municipales.

2. Reconocer los procesos bibliotecarios que se realizan en las bibliotecas municipales.

3. Detallar el estado actual de los servicios y productos de información de las bibliotecas municipales.

4. Determinar los recursos humanos, financieros, bibliográficos y de infraestructura con los que cuentan las bibliotecas municipales.

5. Identificar la infraestructura tecnológica con la que cuentan las bibliotecas municipales.

\section{Objetivo general 2}

Proponer el Sistema Integrado de Gestión Bibliotecaria para el Sistema de Bibliotecas Municipales del cantón Central de San José. 


\section{Objetivos específicos}

1. Determinar los requerimientos funcionales del Sistema Integrado de Gestión Bibliotecaria para la automatización de las bibliotecas municipales.

2. Identificar la factibilidad técnica, económica y operacional para la implementación de un sistema de gestión bibliotecaria para el Sistema de Bibliotecas Municipales.

3. Reconocer los diferentes programas para la automatización de bibliotecas que pueden utilizarse en las bibliotecas municipales.

4. Evaluar sistemas integrados de gestión bibliotecaria para la automatización de bibliotecas, privativos y de acceso libre.

5. Sugerir el sistema integrado de gestión bibliotecaria para la automatización de los servicios y procesos técnico-administrativos del Sistema de Bibliotecas Municipales del cantón Central de San José.

\section{Marco teórico-conceptual}

La revisión de la literatura, permitió abordar diferentes temas, tales como los relacionados a la Información, indicando que ésta se utiliza comúnmente en todas las ciencias, en la vida cotidiana, así como en el desarrollo y evolución de la humanidad; por esta razón, se afirma que es uno de los "principales focos que ilumina nuestra concepción de la vida y del universo en el que nos movemos y que es considerada como materia prima del conocimiento" (García, 1998, p. 305). Lo que significa que se convierte en un componente básico para el desarrollo humano y económico.

En el caso de las bibliotecas, la información se considera un bien económico, la cual en sí misma constituye un sistema de información. En este sentido, un sistema de información es aquel que recopila, administra y manipula un conjunto de datos que conforman la información de una organización. Al respeto, López-Yepes (1997) indica que "partiendo de que la información documental es un proceso comunicativo, el sistema de información aparece enmarcado por tres cuestiones: las necesidades de información, los canales de información y su topología y el concepto de transferencia de información" (p. 52). 
Los sistemas de información se desarrollan e implementan para distintos propósitos, esto dependerá de la organización que requiera usarlos. Respecto a la gestión de los sistemas de información de las bibliotecas municipales, se consideró en la investigación que dichas instituciones no contaban con la tecnología adecuada con todos sus componentes: redes, hardware, software, base de datos, documentos y archivos electrónicos, los cuales son necesarios para el procesamiento de la información que, junto con los sistemas de información, constituyen un sistema dinámico.

Adicionalmente, la revisión de la literatura incluye también algunos aspectos que se relacionan con el tipo de bibliotecas en la que se plantea la propuesta, tales como:

a) Bibliotecas públicas, que según el Manifiesto de la IFLA/UNESCO (2001) se definen como: Una organización establecida, respaldada y financiada por la comunidad. Constituye un centro local hacia el conocimiento y un requisito básico para el aprendizaje a lo largo de los años, para la toma independiente de decisiones, el progreso cultural del individuo y los grupos sociales. (p. 8)

Las bibliotecas públicas juegan un papel importante en el aprendizaje. Su función principal es facilitar el acceso a la información representada en libros y otros materiales de lectura en papel o en formato digital. Son espacios vivos y dinámicos que deben evolucionar paralelamente con los avances tecnológicos y la demanda de servicios de la población usuaria.

b) Bibliotecas públicas de Costa Rica y el Sistema Nacional de Bibliotecas (SINABI) formado por la Biblioteca Nacional y 58 bibliotecas públicas, 33 oficiales y 25 semioficiales, las cuales constituyen centros de información bibliográfica y de extensión cultural que ponen a disposición de los usuarios las obras más representativas del conocimiento universal, desarrollan una labor de fomento a la lectura y difusión de la información de carácter recreativo, informativo y educativo. (Portal del Sistema Nacional de Bibliotecas, SINABI, 2012, sección Acerca del SINABI, párr. 1)

Por otra parte, se incluye información sobre la estructura organizacional del SINABI, indicando que lo forman las siguientes unidades: dirección general, unidad administrativa financiera, unidad técnica, unidad de automatización, taller de restauración y conservación, y la unidad de 
construcción y mantenimiento. Además, gestiona y administra la Agencia Nacional del Número Internacional Normalizado del Libro (ISBN) y el Número Internacional Normalizado de Publicaciones Seriadas (ISSN).

Las bibliotecas públicas de Costa Rica se coordinan y regulan mediante la Dirección General del SINABI, cuyo propósito es favorecer los procesos de reacondicionamiento de la Biblioteca Nacional y la creación de bibliotecas públicas ante los gobiernos locales. Asimismo, se incluyen aspectos de los gobiernos locales, como organización política compuesta por el Concejo Municipal, el Alcalde Municipal y los Concejos de Distritos, cuyo propósito es la prestación de servicios a las comunidades que los conforman. Entre los servicios que se frecen se encuentran los de educación y cultura, en los que se destacan las bibliotecas, la recreación y la cultura.

Además, se incluyen aspectos relacionados con la Municipalidad de San José, con su marco estratégico, objetivos estratégicos, misión, visión, servicios, políticas, actividades, estructura organizacional y dependencias municipales, destacando a la Gerencia de Provisión de Servicios y al Departamento de Servicios Culturales, al cual pertenecen las bibliotecas municipales.

Finalmente, se aborda el tema de la automatización de bibliotecas, indicando, entre otros aspectos que el primer paso en este proceso consistió en la aplicación de la informática para gestionar los procesos básicos, luego se aplicó a los otros aspectos de la biblioteca, incluyendo los servicios que se brindan a los usuarios, sobre todo el de préstamo de material bibliográfico, que era uno de los principales problemas por la necesidad de controlar el material en préstamo, las pérdidas y extravíos de los fondos disponibles en las bibliotecas, por lo que el préstamo fue una de las primeras secciones o procesos que se empezaron a automatizar.

Posteriormente, la Biblioteca del Congreso de los Estados Unidos diseñó un formato de registro llamado Machine Readable Cataloging (MARC), el cual se difundió rápidamente, ya que permitió a las bibliotecas disponer de los registros de esta biblioteca en soporte informático y crear su propio catálogo. Otras de las experiencias en torno a la automatización de bibliotecas surgen cuando varias bibliotecas se agrupan para compartir sus registros, costos y recursos, es así como surge, por ejemplo, el Online Computer Library Center (OCLC). Esta iniciativa permitió 
a las bibliotecas participantes introducir sus datos. Hoy la OCLC es una de las empresas comerciales más reconocidas en todo el mundo.

La automatización de bibliotecas, entonces, se ha convertido en un fenómeno que actualmente es innegable en las prácticas y técnicas organizacionales y de la información. Adicionalmente, se describen, según Chinchilla y Fernández (2012, pp. 5-6), tres niveles de automatización de bibliotecas, entre los que se destaca la automatización de catálogos bibliográficos y los sistemas integrados de automatización de bibliotecas.

El primer nivel se refiere al software especializado en el control bibliográfico, específicamente del módulo de catalogación, es decir, a la automatización de los catálogos bibliográficos y se describen también software que históricamente se han usado en las bibliotecas para la automatización de sus catálogos, tales como Winisis, Isis Marc, Catalis, Clabel y Biblioteca PHP. Por otra parte, en el contexto de los sistemas integrados de gestión bibliotecaria, el concepto de integración puede entenderse como la capacidad multifuncional de los sistemas para integrar todas las funciones necesarias para la gestión de bibliotecas.

La concepción de los SIGB comienza con un esquema modular en el que se incluyen las funciones de circulación, catalogación y adquisiciones. Después, con el surgimiento de estos sistemas de segunda generación fue posible exportar e importar los datos, especialmente bibliográficos basados en el formato MARC. Fue posible también lograr la interconexión entre sistemas, lo que permitió el intercambio de datos provenientes del resto de módulos, hasta la utilización universal de arquitecturas "cliente/servidor" y la incorporación de estándares de intercambio de información como el Z39.50, mediante el cual es posible el acceso e interacción con otros servidores a través de Internet.

En este orden de ideas, se indica que los SIGB han evolucionado hacia lo que se denomina biblioteca 2.0, lo que implica que han incorporado recursos de la web en el módulo del Online Public Acces Catalog (OPAC), lo que permite a las bibliotecas estandarizar servicios y mejorar la consulta de los usuarios, incluso haciendo referencias a foros y redes sociales (Ortega y Saborío, 2015, p. 25). Asimismo, se enlistan software de licencia libre y privativos que se 
enmarcan en este nivel de automatización, como: GNUteca, Koha, Openbiblio, ABCD, SIABUC, Alephino, Aleph 500 y Janinun, destacando sus funcionalidades, requerimientos técnicos, módulos, entre otros.

\section{Metodología}

Este artículo es el resultado del trabajo de investigación aplicada realizado como requisito para optar al título de Maestría Profesional en Bibliotecología y Estudios de la Información con énfasis en Tecnologías de la Información, de la Universidad de Costa Rica. La investigación se realizó en el 2017, los datos se recopilaron en el segundo semestre del 2016 y se contó con el permiso para llevarla a cabo de quien fue la jefa del Departamento de Servicios Culturales, dependencia a la que pertenece el Sistema de Bibliotecas Municipales, así como con el aval del director de Tecnologías de la Información y Comunicación de la Municipalidad de San José.

Adicionalmente, en la investigación se definieron variables para realizar el diagnóstico, lo que permitió describir el estado actual del proceso de automatización de las bibliotecas municipales. Entre las variables que se definieron están: funciones, procesos, servicios (clasificación, descripción bibliográfica, selección de materiales, inventarios) y productos de información, así como los recursos financieros, tecnológicos, humanos, bibliográficos y de infraestructura que se requieren para diseñar la propuesta de implementación del Sistema Integrado de Gestión Bibliotecaria para el Sistema de Bibliotecas Municipales del cantón Central de San José.

El análisis de la información recopilada permitió determinar los requerimientos para la implementación de un SIGB, razón por la cual, la investigación se ubicó desde un enfoque cuantitativo. La población estuvo representada por dieciséis funcionarias de las bibliotecas municipales, organizadas en: seis encargadas de bibliotecas, nueve asistentes del Área de Circulación y Préstamo, una bibliotecóloga del Centro Catalográfico y cuarenta funcionarios de la Dirección de Tecnologías de Información de la Municipalidad de San José. Respecto a las funcionarias de las bibliotecas municipales, se consideró el total de la población, es decir, a dieciséis funcionarias profesionales en bibliotecología que laboran en las siete bibliotecas de la Municipalidad de San José. 
De esta manera, fue necesario obtener una muestra no probabilística para considerar a los funcionarios que laboran en la Dirección de Tecnologías de Información de la Municipalidad de San José, los cuales están representados por tres sujetos, que corresponden al director y los jefes de los departamentos de Soporte Técnico y Desarrollo de Sistemas. Su elección se determinó porque ellos forman parte del grupo de personas en el proceso de toma de decisiones de la Municipalidad de San José.

Entre las fuentes de información que se consultaron están las bibliotecas de la Universidad de Costa Rica y de la Universidad Nacional, en las que se buscaron fuentes primarias y secundarias, tales como libros, monografías, publicaciones periódicas, obras de referencia, literatura gris. También fue necesario investigar fuentes disponibles en Internet. Se consultaron también otras fuentes como documentos internos del Sistema de Bibliotecas Municipales, manuales de procedimientos, políticas internas, plan operativo anual, reglamento Interno y manual de organización de funciones de la Municipalidad de San José. También, fue necesario revisar modelos de evaluación de sistemas de gestión bibliotecaria con el fin de proponer el sistema que cumpliera con los requerimientos y las necesidades de las bibliotecas municipales, así como los servicios que brindan.

Para la recolección de la información se utilizó un cuestionario dirigido a las funcionarias de las bibliotecas municipales que laboran en el Área de Circulación y Préstamo, encargadas de bibliotecas y personal del Centro Catalográfico. El cuestionario se estructuró en seis partes, de acuerdo con los aspectos que se requerían investigar, es decir, recurso humano, generalidades y caracterización de las bibliotecas, recursos financieros, procesos técnicos, infraestructura y recursos tecnológicos. Contenía cuarenta y dos preguntas, treinta y ocho cerradas y cuatro abiertas.

Este instrumento fue valorado y aprobado por las personas encargadas de guiar y dar seguimiento al proceso investigativo dentro de la maestría y se aplicó de manera autoadministrada ya que se envió por correo electrónico y mensajería. Lo respondieron todos los 
sujetos de información, es decir, las dieciséis funcionarias que trabajan en las bibliotecas municipales.

Se aplicaron también entrevistas dirigidas al Director de Tecnologías de Información, a los jefes de los Departamentos de Soporte Técnico y Desarrollo de Sistemas. Para realizar las entrevistas, se diseñaron guías de entrevistas, las cuales permitieron recopilar información técnica y operacional que sirvió como sustento para presentar la propuesta. Estas guías se diseñaron considerando los siguientes aspectos: datos personales, recursos humanos, financieros y tecnológicos de la dirección. La primera guía de entrevista constó de diecinueve preguntas, la segunda de quince y la última incluyó diez preguntas. También, se realizó una investigación de mercado con el fin de recolectar datos de software para la automatización de bibliotecas disponibles en el mercado y buscar proveedores que ofrecían alternativas para dar solución al problema y objetivos planteados en la investigación.

Como resultado de la investigación de mercado, se analizaron los siguientes softwares: KOHA, JANIUM, ALEPH, ALEPHINO y SIABUC 9. Para la evaluación de estas alternativas, se consideraron aspectos tales como: requerimientos generales del sistema, requerimientos de software y hardware, funcionalidades, especificaciones técnicas y operacionales, lo que incluyó viabilidad económica, administrativa, seguridad, facilidad de uso y mantenimiento, proveedor o distribuidor del software a nivel nacional. De cada una de las alternativas se completó una matriz con los datos necesarios. Adicionalmente, se completó una matriz comparativa que permitió describir y evaluar los aspectos técnicos del cada software.

Además, se realizó un sondeo en las bibliotecas universitarias públicas y algunas privadas a nivel nacional que utilizan software de automatización de bibliotecas. Las bibliotecas que se consideraron fueron el Sistema de Información Documental (SIDUNA) de la Universidad Nacional; el Sistema de Bibliotecas, Documentación e Información (SIBDI) de la Universidad de Costa Rica; el Sistema de Bibliotecas del Tecnológico de Costa Rica; la Universidad Autónoma de Centroamérica; la Universidad Latina; la Universidad de Iberoamérica; la Universidad Latinoamericana de Ciencia y Tecnología; el Instituto Nacional de Aprendizaje y la Biblioteca Nacional Miguel Obregón Lizano. 
Para el procesamiento de los datos y la interpretación de la información recopilada con los instrumentos de mediación, se tabuló y los resultados se presentaron mediante gráficos y cuadros elaborados en el programa Excel y Word, lo que permitió la redacción y presentación del documento final. El análisis de los datos se presenta de acuerdo con cada una de las variables definidas en la investigación. Primero se describe el estado actual y el grado de avance en el proceso de automatización de las bibliotecas municipales, servicios y productos, así como los procesos bibliotecarios, recursos humanos, financieros, tecnológicos y de infraestructura.

Con este análisis e interpretación de la información se da respuesta a cada una de las variables y objetivos propuestos, los cuales fueron el insumo principal para la propuesta de implementación del SIGB en el Sistema de Bibliotecas Municipales de la Municipalidad de San José.

\section{Principales resultados}

El diagnóstico permitió determinar lo siguiente:

1. Las bibliotecas municipales tienen un nivel bajo de automatización, ya que en el momento de la investigación solamente contaban con una base de datos bibliográfica diseñada en Winisis, la cual reunía las colecciones bibliográficas de las siete bibliotecas.

2. Desde 2005 las bibliotecas municipales implementaron el uso de Winisis; sin embargo, por tratarse de un software de acceso libre, la Dirección de Tecnologías de Información no brinda ningún tipo de soporte para que las bibliotecas lo siguieran utilizando.

3. Los procesos, tareas y actividades relacionadas con la gestión bibliotecaria se realizaban de forma manual, con el uso de controles que no permitían una gestión más ágil y oportuna.

4. Los procesos de análisis documental, tales como selección y adquisición de material bibliográfico, catalogación descriptiva, clasificación documental e indización, se realizaban de forma manual, mediante el uso de controles que se implementaron desde el Centro Catalográfico.

5. El personal de la Dirección de Tecnologías de Información no tenía conocimiento, ni experiencia en software de automatización de bibliotecas; sin embargo, en caso de 
implementar una solución tecnológica de este tipo, brindarían el apoyo y soporte desde su área de especialización.

6. Las bibliotecas municipales han implementado diferentes servicios y productos de información, tales como talleres, charlas, bibliotecas viajeras, áreas de ludoteca y cómputo, celebración de efemérides, préstamo de material bibliográfico, registro de usuarios, solicitudes de nuevas adquisiciones, entre otros; sin embargo, muchos de estos servicios y productos debían realizarse de forma manual, completando, por ejemplo, boletas que se han diseñado para diferentes propósitos, como la boleta de préstamo a sala y domicilio que el usuario debe completar a mano y que se organizan en un archivo ordenado alfabéticamente.

7. No existía hasta el momento de la investigación un SIGB que permitiera iniciar el proceso de su automatización.

\section{Propuesta}

Para proponer el SIGB que permitiera la automatización de las bibliotecas municipales y como parte del procedimiento, se evaluaron los siguientes softwares: SIABUC9, Aleph, Alephino, Janium y Koha. Entre los criterios que se consideraron para seleccionar los programas fueron: precio, tipo de contratación, aspectos técnicos, operacionales, económicos y de compatibilidad con la plataforma tecnológica institucional. Además, se consideraron los aspectos analizados en el estudio de mercado y el análisis e interpretación de la información recopilada en el diagnóstico. De los programas seleccionados, se analizaron aspectos tales como: datos generales de los proveedores, representante de los proveedores en el país, requerimientos generales del sistema, de software y hardware, tipo de licenciamiento y actualizaciones, soporte y garantía, costo y compatibilidad con la plataforma tecnológica de la Municipalidad de San José.

De cada SIGB se completó una "matriz" y se elaboró un cuadro comparativo con los aspectos técnicos que se tomaron en cuenta para hacer la evaluación. De acuerdo con el análisis de los aspectos técnicos, se logró determinar que cuatro de los SIGB eran compatibles con la 
plataforma tecnológica de la Municipalidad de San José; sin embargo, para la compra de las licencias se consideró el precio de cada uno.

EI ALEPH y ALEPHINO tenían un costo aproximado a los $\$ 60.000 .000$, la diferencia entre ambos es que el ALEPHINO funciona para bibliotecas pequeñas, mientras que el ALEPH es adecuado para bibliotecas más grandes, ambos son distribuidos por la misma empresa. Otro software que se analizó fue el JANIUM, con un costo de más de $\$ 100.000 .000$, precio que sobrepasaba las posibilidades económicas para la compra de licencias. Respecto a KOHA, no fue posible considerarlo debido a que, de acuerdo con los resultados del diagnóstico, se determinó que hasta el momento no existía una política institucional que regulara el uso de software libre y que le permitiera a la biblioteca contar con el visto bueno de la Dirección de Tecnologías de Información, trámite necesario para que el Departamento de Proveeduría y Presupuesto pudiera autorizar la compra.

Según lo anterior y considerando los requerimientos funcionales, técnicos, económicos y operacionales, se propuso implementar ALEPH 500 en el modelo de un servicio en la nube, tomando en cuenta también que dicho sistema, entre otros aspectos, está diseñado con una arquitectura cliente/servidor y utiliza Oracle como administrador de base de datos, el cual era compatible con el gestor de base de datos y con la plataforma tecnológica de la Municipalidad de San José.

Se propuso adquirir un SIGB privativo, debido a que, tal como se pudo determinar en el diagnóstico, el personal de la Dirección de Tecnologías de Información de la Municipalidad de San José no contaba con la experiencia necesaria en el uso de sistemas integrados de gestión bibliotecaria basados en software libre; además, no existían políticas institucionales para la implementación y uso de este tipo de soluciones tecnológicas. La posibilidad de contratar expertos externos para la implementación del sistema tampoco era viable, debido a que tenía que hacerse por servicios profesionales, lo cual no era factible por las políticas institucionales en cuanto a contención del gasto público. 
Se propuso que el proyecto de automatización de estas bibliotecas se gestionara bajo la metodología de administración de proyectos, la cual define, entre otros aspectos, el ciclo de vida de un proyecto; en la Figura 1 se detallan las etapas:

\section{Figura 1}

\section{Propuesta etapas del proyecto de automatización}

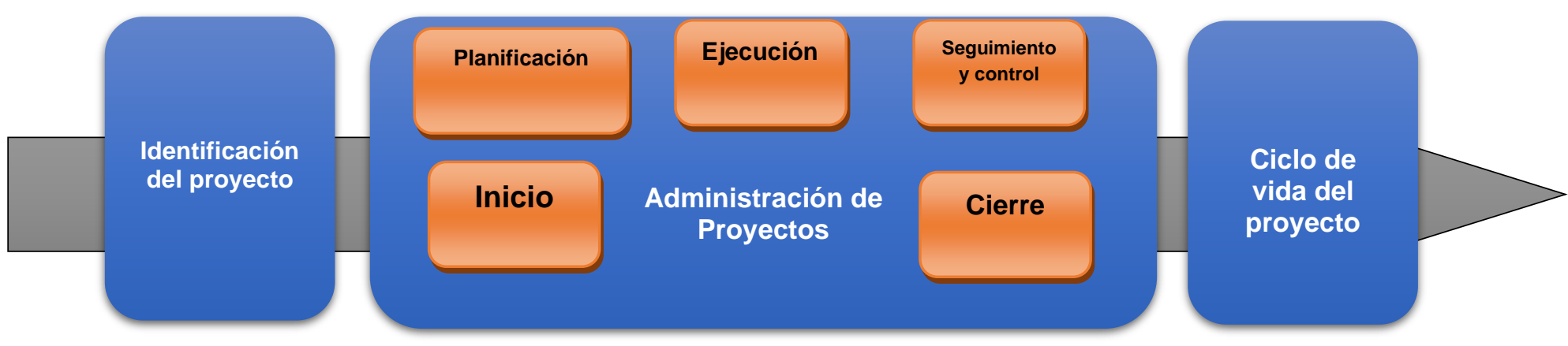

Fuente: Elaboración propia de la autora (2020).

En cada una de las etapas, se definieron las principales actividades y la proyección del tiempo de ejecución (ver Cuadro 1). Asimismo, se detallan los requerimientos funcionales (ver Cuadro 2), la factibilidad económica (ver Cuadro 3), la técnica y operacional para definir los recursos humanos, los financieros y de infraestructura necesaria la implementación del SIGB, como se muestra a continuación:

Cuadro 1. Fases, actividades y proyección de tiempo de ejecución

\begin{tabular}{|l|l|l|}
\hline Etapa & \multicolumn{1}{|c|}{$\begin{array}{c}\text { Actividades } \\
\text { Proyección de }\end{array}$} & $\begin{array}{c}\text { Prempo de } \\
\text { tiempión } \\
\text { ejecución }\end{array}$ \\
\hline Inicio & $-\begin{array}{l}\text { Solicitar a la Gerencia de Provisión de Servicios que se } \\
\text { gestione la propuesta de automatización de bibliotecas } \\
\text { bajo la metodología de administración de proyectos } \\
\text { definida institucionalmente. }\end{array}$ & \\
\hline & $\begin{array}{l}\text { Elaborar el acta de constitución del proyecto, en la cual } \\
\text { se detallan aspectos tales como: nombre del proyecto, } \\
\text { justificación, objetivos, alcance, población beneficiada, } \\
\text { etapas, presupuesto global aproximado, supuestos y } \\
\text { restricciones. }\end{array}$ & 2 meses \\
\hline
\end{tabular}


- Identificar los grupos de interés del proyecto.

- Definir el perfil de los miembros del equipo técnico del proyecto.

- Integrar el equipo técnico del proyecto.

- Nombrar al profesional responsable del proyecto.

- Estructurar detalladamente los paquetes o entregables del proyecto, según las etapas establecidas.

- Enlistar las actividades que se requieren para la gestión del proyecto.

- Solicitar las modificaciones presupuestarias que se requiere para la ejecución del proyecto.

- Gestionar un presupuesto extraordinario para disponer

\section{Planificación}

- Definir el proceso para comunicar los avances del proyecto a los grupos de interés.

- Identificar los posibles riesgos del proyecto y las acciones para su mitigación.

- Convocar a reuniones periódicas al equipo técnico del proyecto. Se propone que estas reuniones se realicen quincenalmente, al menos durante la etapa de planificación, posteriormente pueden realizarse mensualmente.

- Gestionar el acta de inicio del proyecto.

- Verificar que las modificaciones presupuestarias se hayan aprobado.

- Definir claramente los requerimientos y aspectos técnicos del SIGB.

- Elaborar el cartel de licitación para la suscripción en la nube del SIGB, dispositivos, materiales y contrataciones (capacitaciones, migración de datos).

Ejecución - Confeccionar solicitud de contratación (pedido para la suscripción del SIGB).

- Capacitar al personal en los diferentes módulos del SIGB (catalogación, circulación, OPAC, adquisiciones). La capacitación debe incluir:

- Entrega por parte del proveedor del plan de capacitación con cronograma, en el cual deberá definir la modalidad de la capacitación, cantidad de personas, horarios, lugar y equipo necesario.
3 meses

6 meses 
- Preferiblemente, la capacitación debe ser para un mínimo de 25 funcionarios municipales, incluyendo personal de las bibliotecas municipales y de la Dirección de Tecnologías de la Información.

- Entregar anticipadamente los manuales descriptivos en idioma español del funcionamiento de los módulos.

- Realizar la conversión y migración de datos.

- Generar de códigos de barras y acondicionamientos de espacios para instalar computadoras para la consulta del OPAC Web.

- Parametrizar y configurar del SIGB.

- Implementar el Sistema Integrado de Gestión Bibliotecaria.

- Puesta en marcha del SIGB.

- Revisar los paquetes o entregables del proyecto.

\section{Seguimiento} y control

- Realizar reuniones periódicas con el equipo técnico del proyecto.

- Elaborar informes de avance del proyecto.

- Elaborar acta de recepción definitiva del proyecto, que incluye: nombre del proyecto, datos de la empresa contratada y especificaciones de las cláusulas bajo las cuales se recibe el proyecto.

- Elaborar informe final del proyecto, con los siguientes aspectos: nombre del proyecto, nombre del profesional responsable del proyecto y de la persona facilitadora, Cierre cumplimiento de objetivos, satisfacción de los grupos de interés, alcance, balance final sobre la gestión de los 2 meses tiempos de ejecución, así como de la administración de los recursos económicos y humanos, calidad del producto final, logros, limitaciones, riesgos materializados y experiencia en la solución de estos, conclusiones, recomendaciones $y$, si es necesario, fotografías para documentar el informe.

Fuente: Elaboración propia de la autora (2020) 


\section{Cuadro 2. Requerimientos funcionales}

\begin{tabular}{|c|c|}
\hline & Requerimientos \\
\hline Catalogación & $\begin{array}{l}\text { - Creación y mantenimiento de diferentes tipos de recursos bibliográficos, } \\
\text { tales como libros, CD-ROM, mapas. } \\
\text { - Este módulo debe incluir el formato MARC bibliográfico y de } \\
\text { autoridades. } \\
\text { - } \text { Generar diferentes plantillas de captura de datos, según el tipo de } \\
\text { material que se está catalogando. } \\
\text { - } \quad \text { Exportar e importar registros bibliográficos. } \\
\text { - } \quad \text { Creación y modificación de registros. } \\
\text { - } \quad \text { Soportar un número ilimitado de registros. } \\
\text { - } \quad \text { Impresión de etiquetas de códigos de barras y marbetes. } \\
\text { - } \quad \text { Generar diferentes reportes por tipo de material, biblioteca, catalogador. } \\
\text { - } \quad \text { Crear registros con RDA (Recursos, disponibilidad y acceso). }\end{array}$ \\
\hline Circulación & 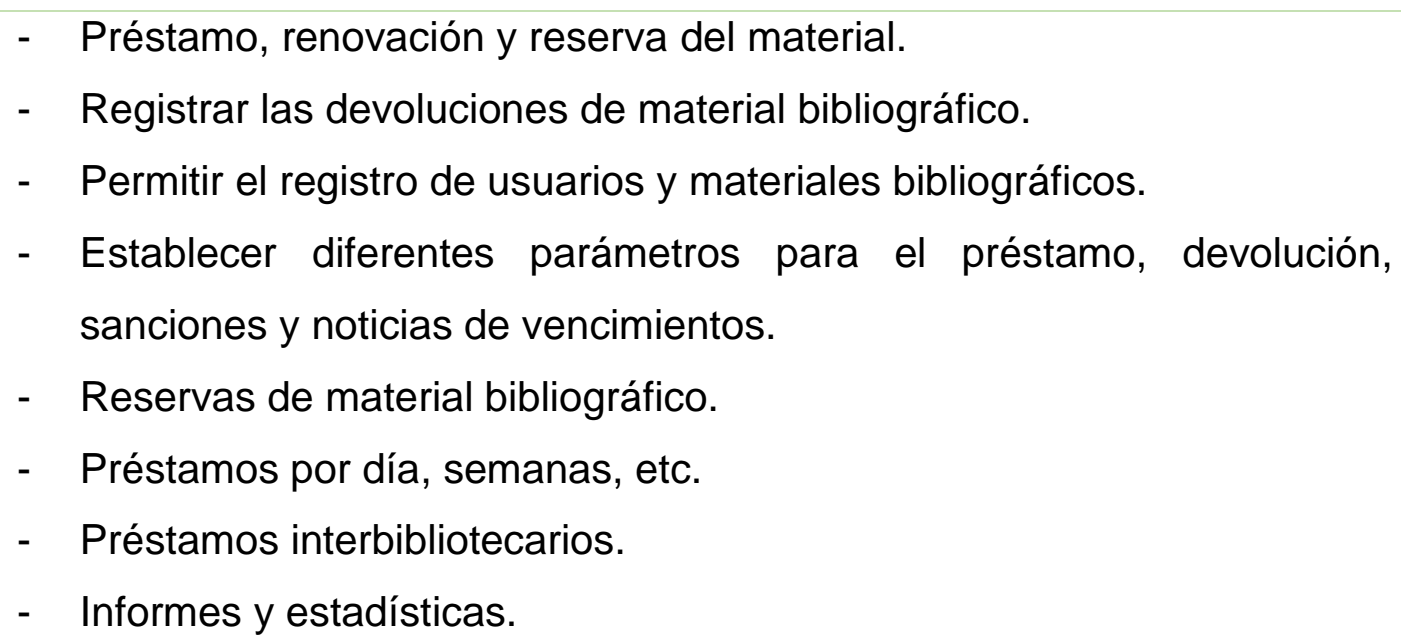 \\
\hline Adquisiciones & $\begin{array}{l}\text { - Gestionar los materiales bibliográficos adquiridos por compra, donación } \\
\text { o canje, el cual debe ser capaz de: } \\
\text { - } \quad \text { Recepción del material bibliográfico. } \\
\text { - } \quad \text { Asignar el presupuesto ordinario y extraordinario para la adquisición del } \\
\text { material bibliográfico. } \\
\text { - Manejar las compras, donaciones y canjes por año de adquisición u otro } \\
\text { tipo de control. }\end{array}$ \\
\hline
\end{tabular}


- Registro del presupuesto, que refleje las cantidades presupuestadas gastadas y comprometidas por biblioteca y el monto disponible para la compra, el gasto y saldos disponibles.

- Solicitudes del usuario o del personal de la biblioteca para la adquisición del material nuevo.

- Descripción bibliográfica del ítem adquirido (precatalogación).

- Permitir el registro de proveedores (nombre, dirección, contacto, correo electrónico, etc.).

- Permitir el manejo de catálogos digitales de los proveedores.

- Generar estadísticas por presupuesto, material adquirido, proveedores, entre otros.

- Consulta vía Web de las colecciones bibliográficas, según las siguientes especificaciones:

- Búsquedas simples y avanzadas.

- Estrategias de búsqueda con operadores boléanos, palabras clave, diccionario, etc.

- Mensajes de ayuda.

OPAC - Disposición de recuperación de datos.

- Acceso a otros catálogos.

- Búsquedas en todas las siete bibliotecas o en una biblioteca en particular.

- Búsquedas en un formato particular o todos los formatos (libros, revistas, CD-ROM).

- Visualización en diferentes formatos de los registros bibliográficos.

Fuente: Elaboración propia de la autora (2020)

\section{a. Factibilidad económica}

Este aspecto se refiere a la inversión económica que se requería para implementar el SIGB. Es importante indicar que entre los resultados del diagnóstico se evidenció que el presupuesto que se invertía en el proceso de automatización de las bibliotecas municipales era relativamente 
poco, considerando que los costos iniciales y de mantenimiento de dicho sistema debía plantearse como un proyecto permanente que debía incluirse en el presupuesto anual de estas bibliotecas. Debido a esto, en el momento de plantear la propuesta, se determinó un presupuesto global aproximado, que se detalla en el Cuadro 3.

\section{Cuadro 3. Presupuesto global aproximado}

\section{Actividad}

Presupuesto

global aproximado

Suscripción anual en la nube de SIGB (Incluye licencia básica, licencias Staff para la gestión de los módulos de: catalogación, OPAC, administración, control de préstamo o circulación, adquisiciones y licencias usuario concurrente para el acceso y consulta del OPAC).

Implementación y servicios, entre los que están la parametrización y configuración del programa y capacitación del personal.

Compra de dispositivos:

- 7 lectores ópticos o scanner bluetooth (se requiere de al menos 1 lector para cada biblioteca)

- 1 impresora para marbetes y códigos de barras

- Etiquetas para marbetes, códigos de barras y cintas de impresora.

Proceso de conversión y migración de datos (se requiere hacer conversión y migración de la base de datos disponible en Winisis al nuevo SIGB.

Fuente: Elaboración propia de la autora (2020)

\section{b. Factibilidad técnica}

Incluye los componentes tecnológicos, tales como software y hardware necesarios para la implantación del SIGB. Se propuso hospedar el sistema como un servicio en la nube o como se conoce en idioma inglés "cloud computing", el cual ofrece características de agilidad, rapidez en su instalación y sobre todo es una modalidad que reduce considerablemente los costos, ya 
que los proveedores deben proporcionar la infraestructura tecnológica necesaria, sin que el cliente deba invertir en la compra de cualquier tipo de hardware y software adicionales. Para instalar ALEPH 500 como un servicio en la nube, las bibliotecas debían contar como mínimo, con los siguientes requerimientos de hardware y software:

- CPU II o superior.

- 512 MB de memoria RAM, preferiblemente 1 GB.

- 10 GB de espacio libre en disco duro.

- Windows 2000, XP, Vista o Windows 7, Windows 10.

- Internet Explorer 6.x o superior, FireFox 2.0 o superior.

- Resolución mínima de monitor: 1024 x 768.

- Mínimo monitor de 17", preferible de 19".

- Permisos de Administrador, durante la instalación y actualizaciones.

- Versiones de 64 bits de Windows.

- Conexión TCP/IP.

- Impresora modelo TTP-244MEPro con su respectivo AC de alimentación eléctrica, con memoria de $4 \mathrm{MB}$, puerto USB 2.0.

- Lectores ópticos o scanner bluetooth con comunicación RF, de 2500 pixeles, con tarjeta de memoria de $256 \mathrm{~K}$, fuente de alimentación de 2 pilas AAA, con cargador y batería para su funcionamiento.

\section{c. Factibilidad operacional}

Este aspecto está relacionado con la necesidad de conformar un equipo interdisciplinario que permitiera planificar y llevar a cabo la propuesta con una visión clara de lo que se requería gestionar. Esto debido a que, según los resultados del diagnóstico, no se contaba, en el momento de realizar la investigación, con un equipo interdisciplinario que se encargara de la gestión del proyecto de automatización. Debido a lo anterior, la propuesta fue contar con un equipo de trabajo conformado por bibliotecólogos e informáticos con la estructura que se presenta en la Figura 2. 
Figura 2. Estructura operacional del proyecto

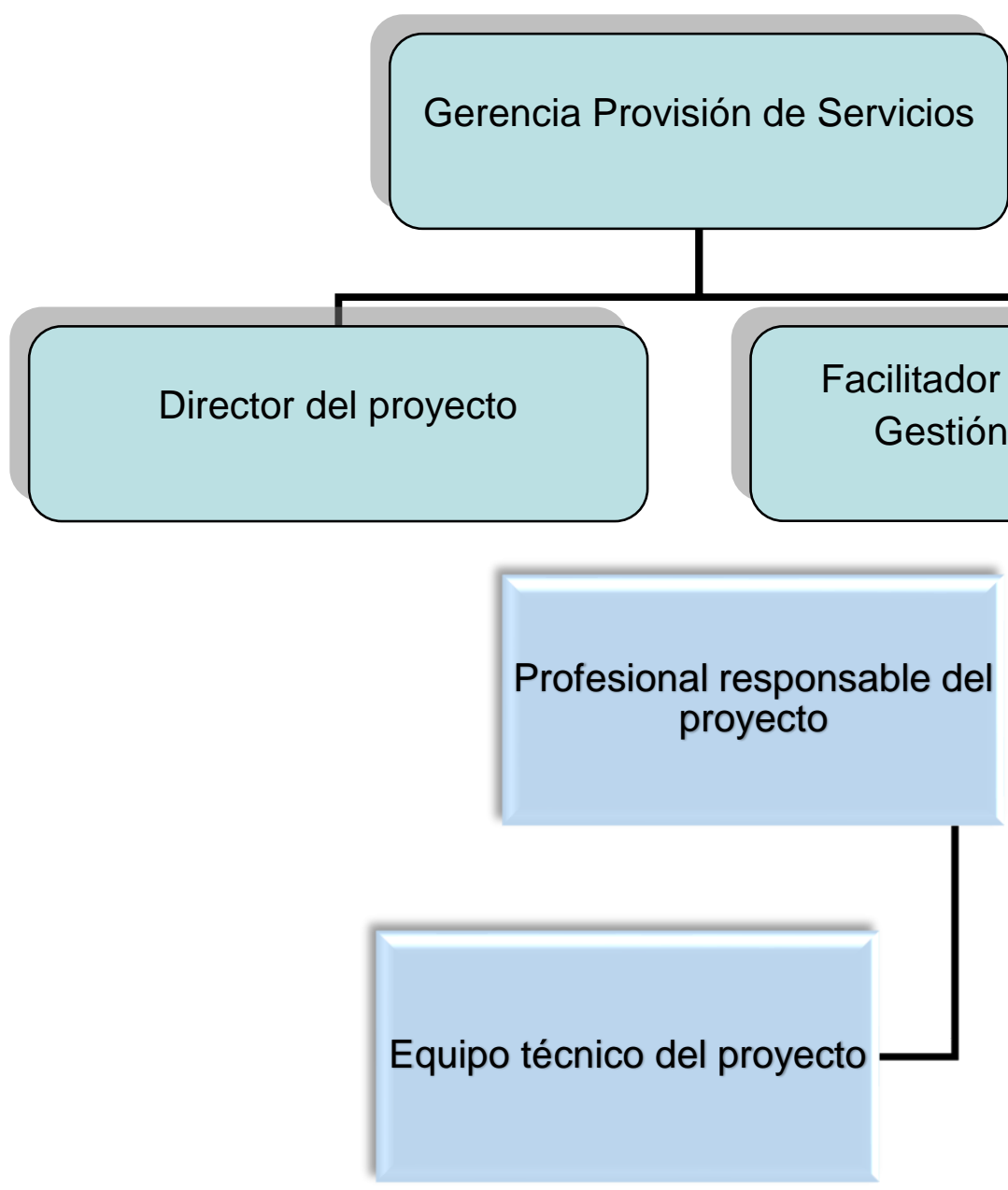

Fuente: Elaboración propia de la autora (2020)

Adicionalmente, se propuso identificar grupos de interés. Estos grupos de interés son las personas o instituciones involucradas en alguna de las etapas de la gestión del proyecto y que se beneficiarían con la implementación del SIGB. Finalmente, dentro de los resultados esperados con la implementación del SIGB están que las bibliotecas municipales logren:

- Disponer del catálogo público en línea para consulta de la población usuaria.

- Automatizar el servicio de circulación y préstamo de materiales bibliográficos.

- Facilitar la gestión de los procesos técnicos y administrativos que se realizan en las bibliotecas.

- Poner en marcha el SIGB con los siguientes módulos: adquisiciones, catalogación, circulación, administración, OPAC. 
- Capacitar al personal en el uso y manejo de los diferentes módulos.

- Conversión y migración de los datos bibliográficos de la base de datos existente al nuevo sistema.

- Préstamos automatizados, gestión y control de multas.

- Control de presupuesto y adquisiciones de material bibliográfico.

- Controles estadísticos, incluyendo los contables.

- Mejoras en los servicios y en la gestión de las colecciones.

- Inventario automatizado de las colecciones disponibles.

- Búsquedas eficientes y pertinentes.

- Registro de usuarios y reportes estadísticos.

- Agilidad en la gestión de las bibliotecas, en sus procesos internos y externos.

- Minimizar tiempos y costos en la gestión bibliotecaria.

- Reducir tiempos de respuestas a las demandas de la población usuaria.

- Ofrecer herramientas a las personas usuarias para que puedan recuperar información de manera rápida y precisa.

- Optimizar el intercambio de recursos y servicios e información institucional.

- Elevar el nivel de competitividad de las bibliotecas municipales mediante la instalación de mejores tecnologías de información y comunicación, en función de las necesidades de los usuarios de nueva generación.

\section{Conclusiones y recomendaciones}

\section{a. Conclusiones}

1. La implementación de un SIGB permitirá que las bibliotecas municipales faciliten a todas las personas, en especial a las del cantón Central de San José, el acceso a la información, a la cultura y al conocimiento.

2. Se considera que la implementación de un SIGB se logrará mejorar la visualización y posicionamiento de las bibliotecas municipales en el contexto y realidad actual. 
3. Al disponer del catálogo colectivo en Internet se logrará poner a disposición de la población usuaria real y de toda aquella que quisiera consultar los recursos y servicios de las bibliotecas municipales.

4. Para implementar un SIGB será necesario tomar en cuenta varios factores fundamentales: los requerimientos mínimos para su ejecución, aplicar estándares bibliotecológicos y formatos bibliográficos existentes, elaboración de manuales de apoyo y tutoriales, servicios a los usuarios del software (foros de consulta, discusión y exposición de inquietudes), facilidad de uso, interfaz, capacitación, migración de datos, costo, procesos de inventarios, préstamos, productividad, compras, donaciones y actualizaciones.

5. Siendo las bibliotecas municipales de la Municipalidad de San José, las instituciones que ofrecen acceso a información y la cultura en el ámbito local, se hace impostergable redefinir tareas, funciones y servicios para estar en correspondencia a las exigencias de la sociedad actual.

6. Es necesario que las bibliotecas municipales planifiquen y desarrollen estrategias que garanticen su lugar en la sociedad como apoyo a los dirigentes locales, miembros de la comunidad local, nacional e internacional.

7. Las bibliotecas municipales, ahora más que nunca son instituciones necesarias que deben contribuir al desarrollo social, cultural y económico de las comunidades donde se ubican.

8. Entre los problemas que resolvería el Sistema Integrado de Gestión Bibliotecaria están: Búsquedas eficientes y pertinentes; Gestión, control de multas, registro de usuarios y reportes estadísticos; Agilidad en la gestión de las bibliotecas, en sus procesos internos y externos; Minimizar tiempos y costos en la gestión; Optimizar el intercambio de recursos y servicios e información institucional; y Agilizar las tareas de la organización bibliográfica, circulación, solicitud de préstamo y control de adquisición de material.

\section{b. Recomendaciones}

1. Aunar esfuerzos del personal de bibliotecas, así como la jefatura del Departamento de Servicios Culturales, para logar la implementación del SIGB.

2. Solicitar al Gerente de Provisión de Servicios, por parte de la jefatura del Departamento de Servicios Culturales, que el proyecto de automatización de bibliotecas se gestione bajo la modalidad de administración de proyectos establecida institucionalmente. 
3. Gestionar jornadas de capacitación para las funcionarias del Sistema de Bibliotecas Municipales, previo a la puesta en marcha del SIGB.

4. Hacer un estudio de las colecciones para definir áreas del conocimiento que contienen y definir debilidades, fortalezas, actualización, soportes, formatos, criterios de selección, descarte y procesamiento documental.

5. Integrar activamente al usuario en el proceso de implementación del SIGB, ya que este generará un cambio significativo en la prestación de servicios.

6. Realizar un análisis de los riesgos del proyecto para definir sus ventajas y desventajas. Esto permitirá identificar los riesgos y tomar las medidas y posibles soluciones.

7. Concientizar a las autoridades municipales para generar compromiso, participación y apoyo al proyecto, especialmente a la jefatura del Departamento de Servicios Culturales, Gerencia de Provisión de Servicios y Dirección de Tecnologías de la Información.

8. Hacer pruebas piloto para explicar el uso y funcionamiento del SIGB a funcionarias y usuarios, así como dar capacitación y actualización al personal de bibliotecas para darle continuidad al proyecto.

9. Realizar actividades promocionales para divulgar el nuevo sistema adquirido por las bibliotecas municipales a los usuarios por medio de talleres, visitas programadas.

10. Divulgar al público en general sobre la adquisición del SIGB por medio de Facebook y el blog del Departamento de Servicios Culturales, Municipalidad, afiches, boletines, página Web institucional, entre otros.

11. Dar a conocer el SIGB a los funcionarios de la Municipalidad por medio de correo electrónico y Muninet.

12. Implementar desde el OPAC Web algunos servicios para integrar la tecnología móvil, por ejemplo, la creación de códigos QR, los cuales permiten leer, desde un dispositivo móvil, como Tablet, teléfonos inteligentes información que los usuarios podrán descargar de forma inmediata, tal como las direcciones de páginas web, perfil de Facebook, entre otros. 


\section{Referencias bibliográficas}

Federación Internacional de Asociaciones de Bibliotecarios y Bibliotecas (2001). Directrices IFLA/UNESCO para el Desarrollo y Servicio de Bibliotecas Públicas. https://www.ifla.org/files/assets/hq/publications/archive/the-public-library-service/pg01-s.pdf

García, F. J. (1998). El concepto de información: una aproximación transdisciplinar. Revista General de Información y Documentación. 8 (1). Recuperado de https://goo.gl/wc2Fcc López-Yepes, A. (1997). Documentación informática: sistemas, redes y aplicaciones. Madrid: Editorial Síntesis.

Ortega, I. y Saborío, J. I. (2015). Desarrollo del módulo para la gestión de adquisiciones en el sistema integrado de gestión bibliotecaria de código abierto ESPABIBLIO (Tesis de maestría inédita). Universidad de Costa Rica.

Saorín, T. (2002). Modelo conceptual para la automatización de bibliotecas en el contexto digital (Tesis doctoral). Recuperada de https://goo.gl/mo6p28

SINABI. (2012). Sistema Nacional de Bibliotecas. Recuperado de http://www.sinabi.go.cr/bibliotecas/index.aspx\#.WGGDAvnhDIU

Torres, M. A. (s.f). Planeación, diseño y desarrollo de bases de datos bibliográficas. Recuperado de https://goo.gl/pr52Cr

Valdés, R. (2007). La biblioteca y los bibliotecarios ante la sociedad de la información y el conocimiento. Acimed 16 (4). Recuperado de https://goo.gl/Eb8DV3

\section{Notas de la autora}

Jenny Ulate Montero: académica de la Escuela de Bibliotecología y Documentación e Información, Universidad Nacional, Costa Rica. Correo electrónico: jenny.ulate.montero@una.cr 